\title{
Communication Challenges for Distributed Teams
}

\author{
Muhammad Asad Bin Khalid \\ Masters in Computer Science Institute of Business Administration Karachi, Pakistan \\ Email: asad.khalid@khi.iba.edu.pk
}

\author{
Asim Farooq \\ Masters in Computer Science, Institute of Business Administration, Karachi, Pakistan \\ Email: asim.farooq@khi.iba.edu.pk
}

\section{Waqas Mahmood}

Department of Computer Science IBA, Karachi, Pakistan

Email: wmehmood@iba.edu.pk

Received: 09 January 2021; Accepted: 25 January 2021; Published: 08 February 2021

\begin{abstract}
The paper discusses the issues and problems faced by individuals working in diverse or distributed teams especially in the software industry. The research shows a feedback and trend of the teams that work in distributed environments letting us understand the behaviors and figuring out solutions for the problems of communication. The trend was already set in a few organizations that provided outsourcing services to their clients but after the recent issue of the pandemic almost all organizations switched towards this trend and started focusing on working in distributed teams and not on site. This ensured the safety of the employees as well as it let them keep their work and performance undisturbed. But in the practice of working in distributed teams the major factor that affected the performance was the problem in communication, either due to the available mediums (online meeting software products) or the internet connections. The only factor affecting the performances somehow was the communication problem factor and we have addressed this in our paper, moreover a survey is also done through a questionnaire with the people of software industries in Pakistan and a result is generated for how the people feel working in distributed environments and what hurdles they faced in communication.
\end{abstract}

Index Terms: Communication, Communication Hurdles, Distributed environment.

\section{Introduction}

In this paper we discussed the problems faced by teams working in a distributed environment locally. By distributed environment it means that the teams are either placed in different regions or the individuals are all separately working from different environments. The following research paper will find out the problems and try to give solutions for them as far as communication of distributed teams is concerned. Working in distributed teams in many cases suitable for companies and also the employs as far as resource mobility and transportation is concerned but the most annoying problem in the process is the communication. Currently we don't have any channel that offers a distortion-less and smooth communication gate-way and this causes delay in the submissions and also affects the overall performance of teams. Different surveys and reviews have already been done on the problems of distributed teams. Distributed teams can have problems for communication either due to the communication channels/tools, language barriers Time zone differences, trust issues and coordination issues. [1] The surveys discuss multiple solutions for the problems in distributed teams. As the paper is about communication hurdles in distributed teams (locally) we will be trying to figure out solutions regarding the tools for having scrum meetings and all the discussions involved in the agile process. The other barriers does not really matter as far as the local market is concerned because we can't have a language barrier as well as no time zone issue. We will be focusing on tools and techniques for better communication with less or no distortion and connectivity issues.

\section{Background}

Distributed teams refer to the group of employees working from different remote areas rather than being located at 
a central physical location/office/headquarter. This has become very common in the last few years specially with the formal introduction of WFH "Work From Home" policy, which was limited only to some western countries before the COVID pandemic, this has started to gain popularity and is becoming a norm even in middle eastern and Asian countries now.

Although the technology is available to connect distributed teams in a matter of seconds and at times it is even more comfortable to talk using the technology when it's a matter of small discussions or a quick yes/no queries rather than leaving your seat and walking up to the other employee. But the disadvantages and limitations still exist and it can't (and may never) be a complete replacement of physical interactions.

The goal for this paper is first to identify the hurdles faced by distributed QA teams. We'll be studying and going through the research work already done on this topic so we don't actually go into re-inventing the wheel. We'll be doing our own survey and based on the responses will be compiling the result. We'll be targeting the IT professionals with special attention to the Developers and SQA teams.

\section{Literature Review}

In this literature review, we'll be reviewing and sharing views on various sources presented in the resource section on the topic i.e. communication hurdles in distributed teams or the teams working in distributed environment. For the literature review an approach suggested by the supervisor was used where first different journals database were searched for specific topic using the keywords. Then the topics shown as the result were skimmed (abstract section) to see if these were of our interest. Those which were found to be relevant were downloaded and read in full to develop our understanding on this. So, these were the three steps used for the literature review. Also, referred in the paper "A Survey of Pakistan's SQA Practices: a Comparative Study”.

1. Keyword searching in journal database.

2. Paper review by skimming through the abstract section.

3. Detailed study of the paper.

Although we did not use too many journals for the research but following were the two which were mainly used and had enough material on this topic.

\section{A. Objective}

To review the research done on the topic "communication hurdles between distributed teams" using multiple research papers, develop understanding on the topic, make and conduct a survey based on our own questionnaire, collect responses and share the findings.

\section{B. Overview}

Coordination decrement is the term used by Stephen M. Fiore (2003) which he refers as the invariant of difficulty arising when all members attempt to work together at their full potential (Steiner 1972). He further adds that we must acknowledge that coordination decrement may occur to a much larger degree with distributed teams and teams of teams (Fiore et al. 2001a). In absence of co-location the characteristics associated with interaction the coordination decrement increases in the distributed teams.

On one hand distributed teams adds value to the organization in meeting the goals team interaction with distributed teams creates a lot of issues not only with team's performance but individual performance as well. Co-location provides visual cues about the team mates action which is not the case in distributed teams.

Stephen M. terms this as Team Opacity i.e. a phenomenon where teams members must deal with an increased level of abstraction forced upon them by their distributed nature. According to him the awareness of team member actions decreases due to distribution. It's actually due to change in data from rich environment to a data lean and cognitive environment (An environment where formerly perceptual cues are supplanted entirely or interpreted via, for example, video media).

Another research conducted on communication and coordination in collaborative software development where the evaluation of the qualitative comments was performed by the authors, their results indicated that communication and coordination problems can arise if not properly managed among the teams but at the same time, team can also work effectively despite of lean communication. It stated that members using the lean communication mode are more prone to problems and can break communication with their team members.

\section{Methodology}

The methodology we are following for this research is a questionnaire based survey. The results and hypothesis are given after the responses from public related to the field were gathered. The target audience for this research are usually the Software quality engineers or either at least anyone from software industry who have been working in a distributed 
environment especially during the covid-19 lockdowns. A questionnaire is provided to each participant of the survey which is read and answered by the participants as per the experience of them working in distributed teams. The questionnaire was partially custom and partially adapted from an already published paper.

In the end an opinion based question is also kept to know the end results of them as far as working in distributed environments is concerned. The results were then collected and analyzed to make sure what the experience of all the participants is and what they think regarding working in distributed environments.

\section{Results}

The results are made on the basis of responses gathered from the public after distributing the survey questionnaire. The results are graphically displayed to show the responses from the people.

We received 111 responses altogether from different people working in different field of work, majority of them were from the software industry. As shown in fig. 1.

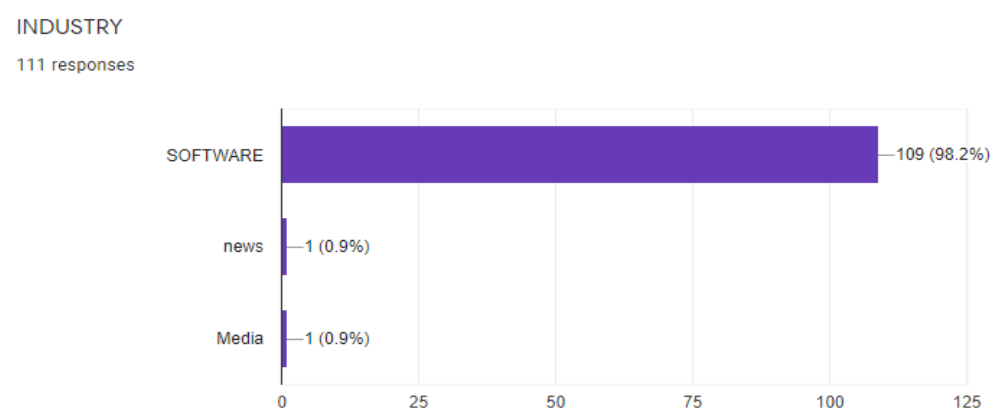

Fig.1. Industry of Respondents with Percentages

The responses received for the questions start from fig. 1.2 and go on respectively.
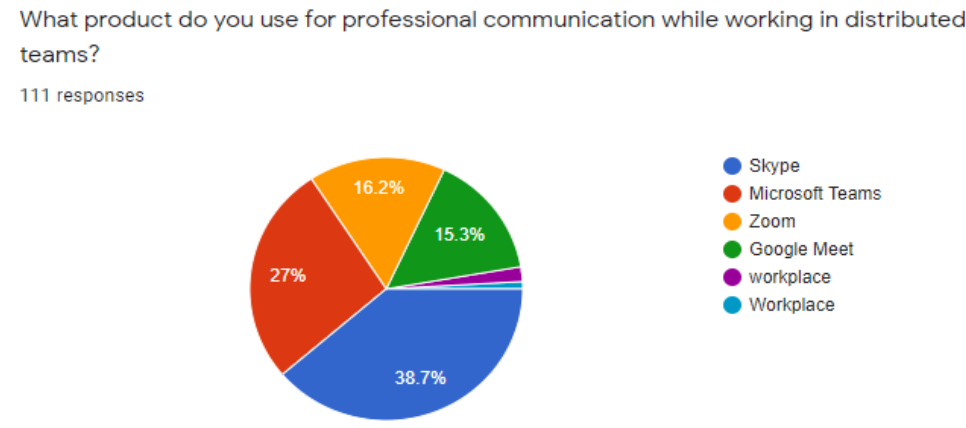

Fig. 2

The first question was related to the product used by individuals for communication purpose while working in distributed teams. $38.7 \%$ of the responders used skype as a tool for communication. $27 \%$ used Microsoft teams, $16.2 \%$ of the total responders were using Zoom while $15.3 \%$ used Google Meet.

Teams are not available at the same time while working in distributed teams

111 responses

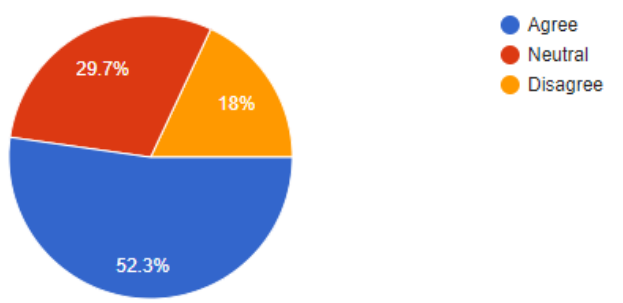

Fig. 3 
The second question was related to time management, which asked the responders that do they think teams are not available on time? 52.3\% agreed to the statement. 29.7\% stayed neautral which mean they neither agreed nor disagree, whereas only $18 \%$ disagreed the clause.
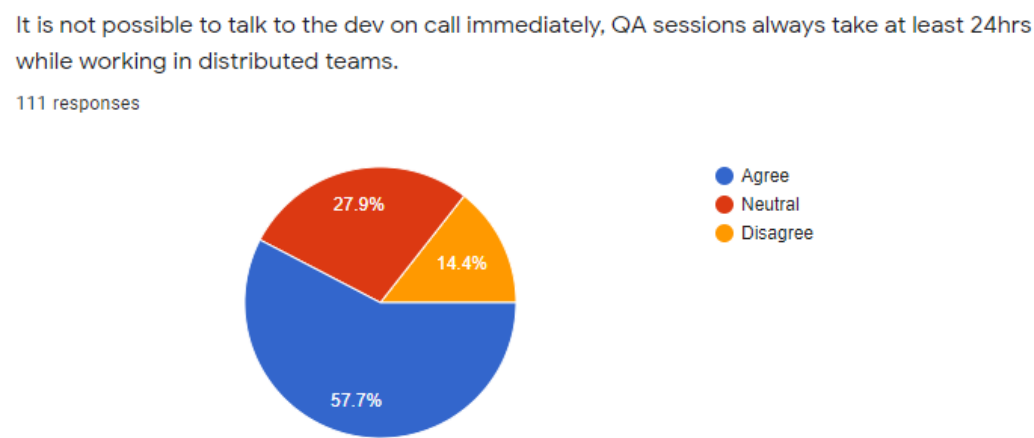

Fig. 4

The third statement stated that QA sessions take almost 24 hours due to communication issues with development team. $57.7 \%$ agreed to this statement. $27.9 \%$ stayed neautral while $14.4 \%$ disagreed.

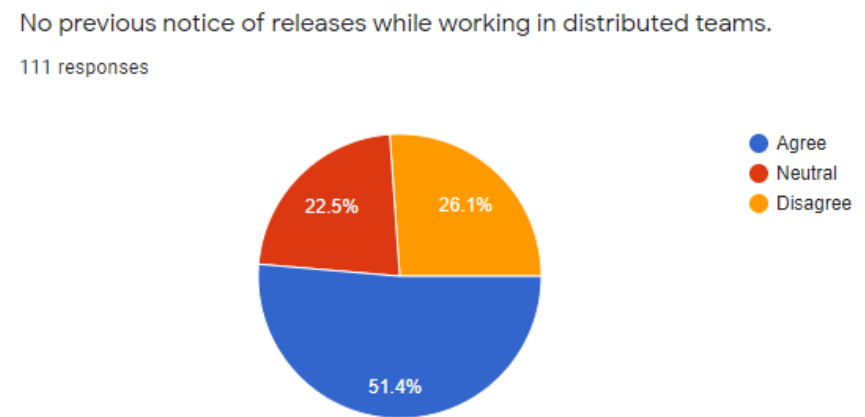

Fig. 5

The fourth statement stated, there was no previous notice of releases. 51.4\% agreed to this statement. $22.5 \%$ stayed neautral while $26.1 \%$ disagreed.

A team does not know the routine of another while working in distributed teams.

111 responses
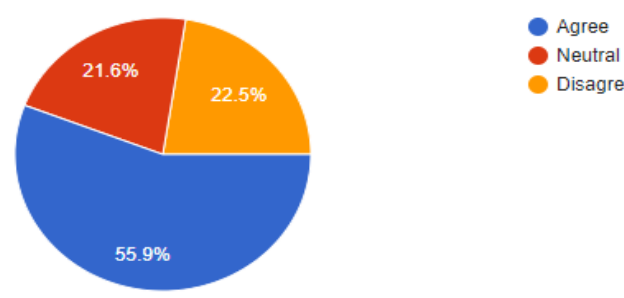

Disagree

Fig. 6

The fifth statement stated, that teams didn't know routine of other teams. $55.9 \%$ agreed to this statement. $21.6 \%$ stayed neautral while $22.5 \%$ disagreed 
Lack of prioritization of bugs open by ops while working in distributed teams.

111 responses

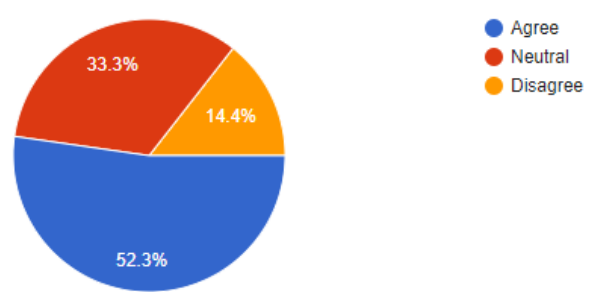

Fig. 7

The sixth statement stated, bugs opened by ops lacked prioritization. $52.3 \%$ agreed to this statement. $33.3 \%$ stayed neautral while $14.4 \%$ disagreed
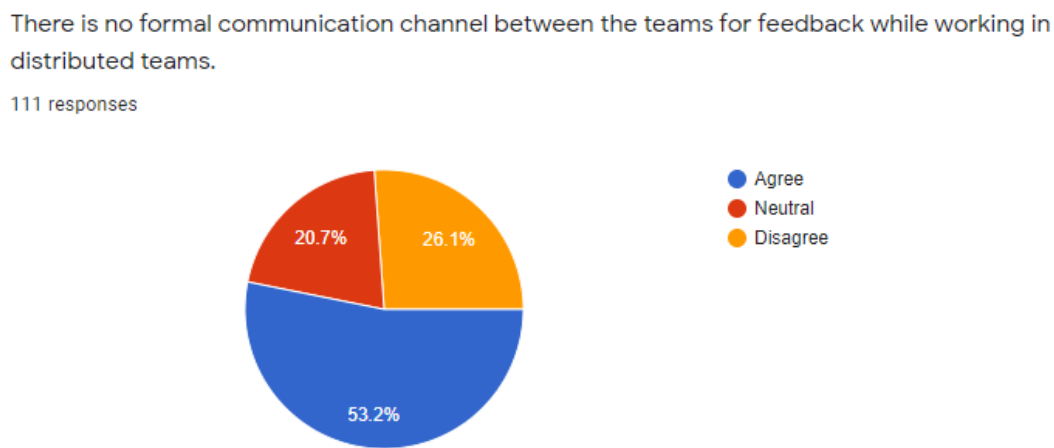

Fig. 8

The seventh statement stated, there was no formal communication channel for feedbacks while working dispersely. $46.8 \%$ agreed to this statement. $28.8 \%$ stayed neautral while $24.3 \%$ disagreed

Dispersed teams are more productive.

111 responses

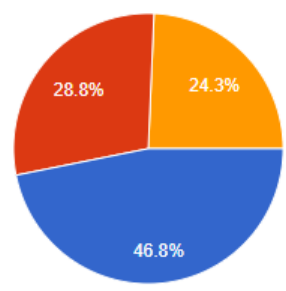

Fig. 9

Quality of communication does get affected when it hap-pens over internet rather than face

to face.

110 responses

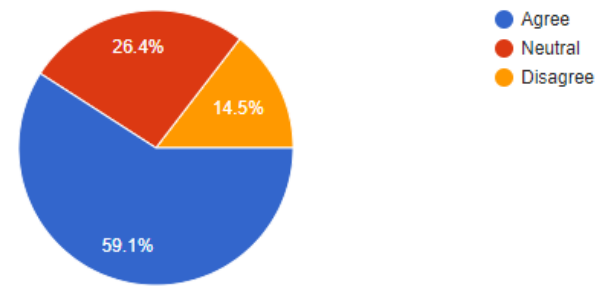

Fig. 10 
Online communication is more comfortable than physical or face to face communication.

110 responses

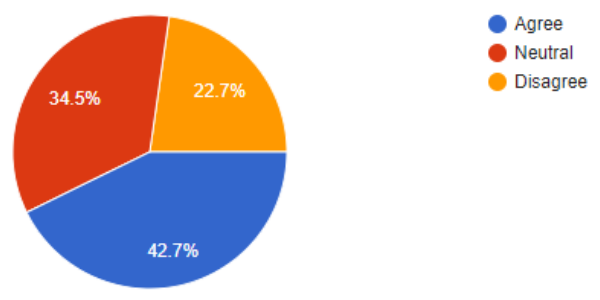

Fig. 11

Clarity in communication lacks when it occurs over any online channel,

110 responses

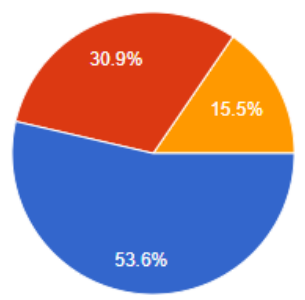

Agree

Disagree

Fig. 12

Responsiveness is a major concern in online communication.

110 responses

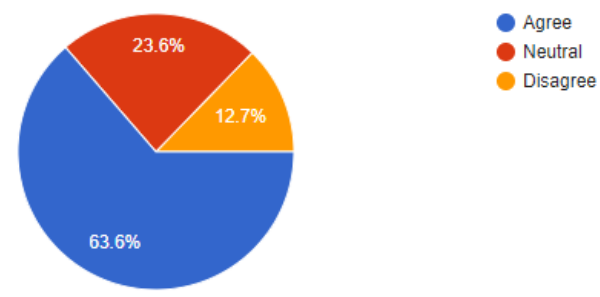

Fig. 13

The results revealed that most of the people thought that dispersed or distributed teams are not that effective, while some of them had a neutral response towards the question which means they had no opinion for that specific question. Moreover the communication clarity and response towards working face to face was also quite similar that majority of the reviews said that clarity in communication lacks in online medium.

\section{Conclusion}

The paper was targeted to highlight the problems faced by individuals while working in distributed environments. Issue that caused this major shift for all companies to implement working in dispersed teams was the recent pandemic. We tried focusing on highlighting the communication hurdles the teams usually face while working as dispersed teams. It could either be a communication between members of a same team or communication with the members of different units such as dev and SQA teams. Issue that arose while working dispersedly was the dependency on others caused due to communication gap either due to the mediums available for managing the meetings or because of the quality of internet available locally. As we focused only on the local scale our survey and its results are also targeted towards the local audience or the local software industry individuals. As described in the results it is quite visible that most of the responders were not in favor of working in dispersed teams, the numbers in result show that majority people were convinced that communication over and online channel is not that much affective. Moreover different other issues were also highlighted through the survey, a time gap or a time mismatch was also a considerable factor which affects the performance of teams and the organizations as well, $52.3 \%$ of the responders agreed to the point that teams are not available on same time. As far as SQA is concerned, the bug reporting also gets affected just because of these 
communication hurdles, $57.7 \%$ of the responders for the question related to SQA were agreed that it takes almost 24 hours to complete a QA session.

The future work for the research includes other cultural diversities to be discussed for the hurdles in communication by the teams in distributed environment. The approach for the future work involves targeting the international community rather than targeting the local software industry. Although the survey was based on the hurdles faced in the communications between distributed teams but were more focused on the channels used for the communications and the problems associated or surfacing due to use of those channels.

During all this we intentionally avoided touching the soft skills and cultural diversities of the distributed teams. There can be a separate list of issues that may arise (or at least shall be considered) due to the cultural differences among the distributed teams. The cultural difference topic itself is a quite dynamic in nature and need a separate survey just to cover the specific issues related to it when it comes to distributed team's communication.

In the future work related to this survey we can touch the issues arising due to the cultural diversities among the distributed teams. We may even take specific cultures which are quite far from each other not only geographically but in customs and traditions as well, say America and Japan.

We have already shortlisted a couple of research papers e.g. "Real-time collaborative editing behavior in USA and Japanese distributed teams DOI: 10.1145/1978942.1979109" looking at how distributed teams from cultural differences are collaborating over real time editing tools. E.g. Live Deck. Difference in behavior and attitude of team members were uncovered using such research papers and we may work on the same patterns.

Further, not only the communication between peers or colleagues from two different cultures shall be considered but we shall also try to include or consider communications between managers and their sub ordinates where both the manager and sub ordinate belong to a different culture. Similarly, the survey can also touch the effect of cultural differences on the outcomes of communication specially when there is a negotiation going on.

To conclude it can be said that working dispersedly is not bad as it allows some flexibility to the workers as well as to the employers but the only thing affecting is the communication gap caused due to multiple reasons. The gap once fulfilled could result in a very productive approach.

\section{Annexure}

Survey Questionnaire: The survey questionnaire was partially adapted as Q2-Q7 were taken from an already published paper named "Communication challenges and strategies in distributed DevOps"[15]. Rest of the survey questionnaire consisted of questions that were self-made as per the topic of the research. Following is the compiled questionnaire that was distributed:

Q1. What product do you use? [14]

- Skype

- Microsoft Teams

-Zoom

- Google Meet

- Other

Q2. Teams are not available at the same time [15]

- Agree

- Neutral

- Disagree

Q3. It is not possible to talk to the dev on call Immediately, QA sessions always take at least 24hrs [15]

- Agree

- Neutral

- Disagree

Q4. No previous notice of releases [15]

- Agree

- Neutral

- Disagree

Q5. A team does not know the routine of another [15]

- Agree 
- Neutral

- Disagree

Q6. Lack of prioritization of bugs open by ops [15]

- Agree

- Neutral

- Disagree

Q7. There is no formal communication

channel between the teams for feedback [15]

- Agree

- Neutral

- Disagree

Q8. Dispersed teams are more productive

- Agree

- Neutral

- Disagree

Q9. Quality of communication does get affected when it happens over internet rather than face to face.

- Agree

- Neutral

- Disagree

Q10. Online communication is more comfortable than physical or face to face communication.

- Agree

- Neutral

- Disagree

Q11. Clarity in communication lacks when it occurs over any online channel.

- Agree

- Neutral

- Disagree

Q12. Responsiveness is a major concern in online communication.

- Agree

- Neutral

- Disagree

\section{References}

[1] Walimbe, P. (2016).To Overcome Communication Challenges in Distributed/Virtual Scrum Teams.

[2] Communication in Distributed Agile Development: A Case Study Mikko Korkala and Pekka Abrahamson VTT Technical Research Centre of Finland.

[3] Quality Issues in Global Software Development Sanjay Misra Dept. of Computer Engineering, Atilim University Ankara, Turkey . Fernández-Sanz Dept. of Computer Sciences, Universidad de Alcalá Alcalá de Henares,

[4] Farooqui Sumeen, and Waqas Mahmood. "A Survey of Pakistan's SQA practices: A Comparative Study." 29th International Business Information Management Association Conference. 2017.

[5] Zia, Ahmed, Waleed Arshad, and Waqas Mahmood. "Preference in using agile development with larger team size." International Journal of Advanced Computer Science and Applications 9.7 (2018): 116-123.

[6] Usmani, Nabiha, Rabbia Hassan, and Waqas Mahmood. "Impediments to Requirement Engineering in Distributed Team." International Journal of Information Engineering and Electronic Business 9.6 (2017): 10.

[7] Stephen M. Fiore, Eduardo Salas, Haydee M. Cuevas \& Clint Bowers (2003) Distributed coordination space: Toward a theory of distributed team process and performance, Theoretical Issues in Ergonomics Science, 4:3-4, 340-364, DOI: 10.1080/1463922021000049971

[8] David J. Pauleen (2003) Lessons Learned Crossing Boundaries in an ICT-Supported Distributed Team, Journal of Global Information Management (JGIM) 11(4) DOI: 10.4018/jgim.2003100101

[9] M. Farmer (2004) DecisionSpace infrastructure: agile development in a large, distributed team Agile Development Conference DOI: 10.1109/ADEVC.2004.11

[10] Pamela Hinds, Sara B. Kiesler, Sara Kiesler. Distributed Work MIT Press 2002 Business \& Economics 
[11] Fatma Cemile Serçe, Kathleen Swigger, Ferda Nur Alpaslan, Robert Brazile, George Dafoulas, Victor Lopez, Online collaboration: Collaborative behavior patterns and factors affecting globally distributed team performance, Computers in Human Behavior, DOI: 10g.1016/j.chb.2010.09.017

[12] Kenton O'hara, Jesper Kjeldskov, Jeni Paay (2011) Blended interaction spaces for distributed team collaboration DOI: $10.1145 / 1959022.1959025$

[13] Khan, Arif \& Keung, Jacky \& Hussain, Shahid \& Bennin, Kwabena. (2015). Effects of Geographical, Socio-cultural and Temporal Distances on Communication in Global Software Development during Requirements Change Management: A Pilot Study. ENASE 2015 - Proceedings of the 10th International Conference on Evaluation of Novel Approaches to Software Engineering. DOI: 10.5220/0005331401590168

[14] Layman, L., Williams, L., Damian, D., \& Bures, H. (2006). Essential communication practices for Extreme Programming in a global software development team. Information and software technology, 48(9), 781-794.

[15] Diel, E., Marczak, S., \& Cruzes, D. S. (2016, August). Communication challenges and strategies in distributed DevOps. In 2016 IEEE 11th International Conference on Global Software Engineering (ICGSE) (pp. 24-28). IEEE.

[16] Liu, L. A., Chua, C. H., \& Stahl, G. K. (2010). Quality of communication experience: Definition, measurement, and implications for intercultural negotiations. Journal of Applied Psychology, 95(3), 469-487. DOI: 10.1037/a0019094

[17] Adler, N. J., \& Graham, J. L. (1989). Cross-cultural interactions: The international comparison fallacy. Journal of International Business Studies, 20, 515-538.

[18] Kenneth L Hacker \& Robert Steiner (2009) Hurdles of access and benefits of usage for internet communication. DOI: $10.1080 / 08824090109384821$

[19] M Pikkarainen, J. Haikara, O. Salo, P. Abrahamsson \& J. Still (2008) The impact of 9agile practices on communication in software development. DOI: 10.1007/s10664-008-9065-9

[20] Viktoria Stray, Nils Brede Moe (2020) Understanding coordination in global software engineering: A mixed-methods study on the use of meetings and Slack. DOI: 10.1016/j.jss.2020.110717

[21] Cripe Krisda. Leading and Communicating to Virtual Teams by IT Project Managers. ProQuest Dissertations Publishing, 2020. 27740504.

[22] DJ Pauleen, P Yoong (2001) Facilitating virtual team relationships via Internet and conventional communication channels. DOI: $10.1108 / 10662240110396450$

[23] Oussama Saafein and Gholam A. Shaykhian (2013) Factors affecting virtual team performance in telecommunication support environment. DOI: 10.1016/j.tele.2013.10.004

[24] Ian Gorton and Sanjeev Motwani (1996) Issues in co-operative software engineering using globally distributed teams. DOI: 10.1016/0950-5849(96)01099-3

[25] Pamela J. Hinds and Mark Mortensen (2005) Understanding Conflict in Geographically Distributed Teams: The Moderating Effects of Shared Identity, Shared Context, and Spontaneous Communication. DOI: 10.1287/orsc.1050.0122.

[26] Reza Barkhi, Ali Amiri \& Tabitha L. James (2014) A Study of Communication and Coordination in Collaborative Software Development. DOI: 10.1080/1097198X.2006.10856414

[27] Lauren E Scissors, N. Sadat Shami, Tatsuya Ishihara, Steven L. Rohall, Shin Saito (2011) Real-time collaborative editing behavior in USA and Japanese distributed teams DOI: 10.1145/1978942.1979109

\section{Authors' Profiles}

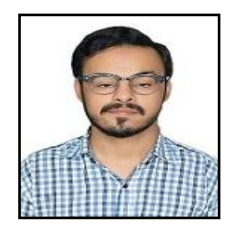

Muhammad Asad Bin Khalid, Masters in Computer Science Institute of Business Administration Karachi, Pakistan asad.khalid@khi.iba.edu.pk

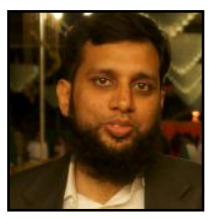

Asim Farooq, Masters in Computer Science Institute of Business Administration Karachi, Pakistan asim.farooq@khi.iba.edu.pk

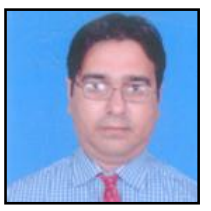

Waqas Mahmood, Department of Computer Science IBA, Karachi, Pakistan, wmehmood@iba.edu.pk 
How to cite this paper: Muhammad Asad Bin Khalid, Asim Farooq, Waqas Mahmood, " Communication Challenges for Distributed Teams ", International Journal of Engineering and Manufacturing (IJEM), Vol.11, No.1, pp. 19-28, 2021. DOI: 10.5815/ijem.2021.01.03 